\title{
Design and Analysis of Mechanism Based on Topological Theory
}

$$
\text { Jian-Guo LUO }{ }^{1} \text {, Jian-You HAN² }
$$

\author{
${ }^{1}$ North China Institute of Science and Technology, Mechanical \& Electrical Department, Beijing, \\ 101601 \\ ${ }^{2}$ Beijing University of Science \& Technology, School of Mechanical Engineering, Beijing, 100083 \\ luojg_1598@163.com
}

\begin{abstract}
Keywords: Topology, Graph theory, Moving capability, Degree of freedom, Design.
\end{abstract}
\begin{abstract}
Based on the analysis of current developing state of graph theory, define the description of spatial moving capability of common couples and translation base and rotation base of mechanism, based on the fact of mechanism consists of components, a new description method by topological graph theory of components relationship suggested, new description of serial mechanism and parallel mechanism and hybrid mechanism obtained with this method, description elements include component pane and constrained component pane and component relationship line and constrained component relationship line and spatial relative moving capability between adjacent components. DOF(degree of freedom) and working space of serial mechanism and parallel mechanism and hybrid mechanism obtained based on the describing method and calculation method of branch spatial moving capability. Inverse design method suggested with object moving capability and level of working space and accuracy and loading capability, according to the different twelve type of object goal, three hundred and twenty four types of mechanism presented with judging condition.
\end{abstract}

\section{Introduction}

Research on the system of mechanism kinematic chain already had hundred years history, in 1964, Freudenstein and Dobrjanskyj[1] first introduced graph theory of mechanism, which used to represent the topological structure of the kinematic chain. With the help of graph theory[2], number of kinematic chains with various component number and moving pair number have been integrated out, these kinematic chains can be transformed into more different mechanisms, which greatly enriches the structure types of planar mechanism, graph theory is introduced to express the topological structure of kinematic chains has brought great convenient to the study of mechanism, and opens up a new way for the mechanism research. At the same time, the topological map can be expressed with matrix such as adjacency matrix, correlation matrix [3] and so on, while matrix is convenient for mathematical analysis and treatment, graph theory provides a powerful mathematical tool for the research and development of mechanism structure, it also greatly accelerated the conceptual design of automatic machine based on computer, thus forming the new direction of research on basic theory of mechanism. The calculation of degree of freedom of mechanism is originally a simple question, correct results can be obtained by using the traditional Kutzbach-Grübler formula [4-6], only on the basis of arithmetic calculation. However, in the course of the development of mechanism, many examples do not meet the above formula[7-8], Huang Zhen apply the screw theory to deal with this problem, constraint screw presented[9] to find the degree of freedom of mechanism. However, the above methods can only provide abstract analysis of the degrees of freedom of mechanism, a new method based on graph theory is proposed in this paper, intuitive analysis can be carried out then, still singularity may occurred can be also be analyzed then, great significance displayed even in the solving of working space in future.

\section{Description with Graph Theory of Mechanism}

\section{Spatial Moving Capability of Pair and the Moving Base}

Rotating pair, translation pair, sphere joint, hooke joint are common motion pairs, they are one 
dimensional, two dimensional, three dimensional, one dimensional spatial moving capability, because the each motion pair consists of two adjacent components[10], relative motion range of two adjacent components in space is limited, as shown in figure 1 to 4.

$\mathrm{P}\left(n_{i}, d_{n_{i}}\right)$ is defined as the spatial moving capability of translation pair between two adjacent components(as shown in Figure 1), $n_{i}$ is the direction of guide component, $d_{n_{i}}$ is the difference between limited moving distances of mobile component along the guide component.

$\mathrm{R}\left(n_{i}, \alpha_{n_{i}}\right)$ is defined as the spatial moving capability of rotation pair between two adjacent components(as shown in Figure 2), $n_{i}$ is the direction of rotation axis, $\alpha_{n_{i}}$ is the difference between limited rotating angles of one component rotating relative to another.

$\mathrm{T}\left(n_{i}, \alpha_{n_{i}} ; n_{j}, \alpha_{n_{j}}\right)$ is defined as the spatial moving capability of hooke joint between two end components(as shown in Figure 3), $n_{i} 、 n_{j}$ are the direction of two rotation axis, $\alpha_{n_{i}} 、 \alpha_{n_{j}}$ are the difference between limited rotating angles of one component rotating relative to another separately.

$\mathrm{S}\left(n_{i}, \alpha_{n_{i}} ; n_{j}, \alpha_{n_{j}} ; n_{k}, \alpha_{n_{k}}\right)$ is defined as the spatial moving capability of sphere joint between two end components(as shown in Figure 4), $n_{i}, n_{j}, n_{k}$ are the direction of two rotation axis, $\alpha_{n_{i}}, \alpha_{n_{j}} 、 \alpha_{n_{k}}$ are the difference between limited rotating angles of one component rotating relative to another separately.

$\operatorname{trans}\left(n_{i}, s p_{0}, d_{n_{i}}\right)$ is defined as the translation base, $\operatorname{rot}\left(n_{i}, s a_{0}, \alpha_{n_{i}}\right)$ is defined as the rotation base, $n_{i}$ is the axis of translation or rotation, $s p_{0}$ and $s a_{0}$ are the initial point of moving and initial rotation angle separately, $d_{n_{i}}$ and $\alpha_{n_{i}}$ are the moving distance along moving axis $n_{i}$ and rotation angle around the rotation axis $n_{i} \cdot \operatorname{trans}\left(n_{i}, s p_{0}, d_{n_{i}}\right)$ and $\operatorname{rot}\left(n_{i}, s a_{0}, \alpha_{n_{i}}\right)$ can be $\operatorname{simplified}$ as $\operatorname{trans}\left(n_{i}\right)$ and $\operatorname{rot}\left(n_{i}\right)$ or $\operatorname{trans}\left(n_{i}, d_{n_{i}}\right)$ and $\operatorname{rot}\left(n_{i}, \alpha_{n_{i}}\right)$ in describing the spatial moving capability of mechanism.
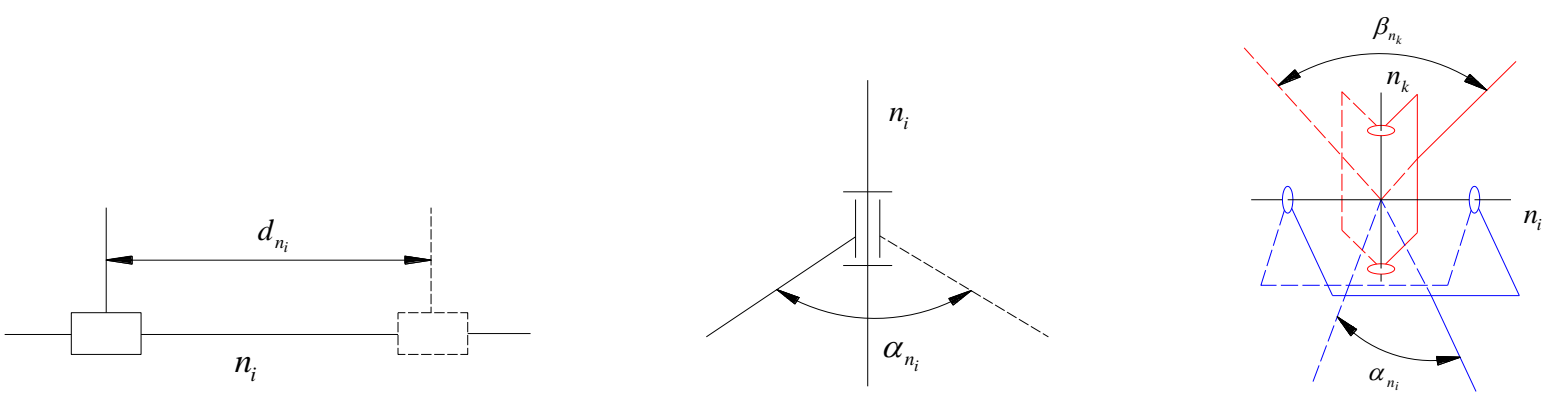

Fig. 1 Spatial moving capability Fig. 2 Spatial moving capability Fig. 3 Spatial moving capability of translation pair of rotation pair of Hooke joint

\section{Description in Graph Theory of Components Relationship of Mechanism}

Kinematic pair connected according to certain spatial structure and layout, serial mechanism composed of single transmission path and single output (as shown in Figure 5), parallel mechanism composed of multi transmission paths and single output(as shown in Figure 6), hybrid mechanism composed of multi transmission paths and main output and auxiliary output as well(as shown in Fig. 7). How to use the method of graph theory to describe this different types of mechanisms, as well as the degrees of freedom and singularity of mechanism carried out, a new description method of graph theory presented here.

Based on the fact that mechanism is composed of a lot of components connected orderly, executive component and fixed component contained in each mechanism, which are defined as $G$ and $\mathrm{H}$, the remaining components named in Arabic numerals, each component expressed by a blocked number, shown as $\mathrm{G}$ and $\mathrm{H}$ in Figure 8, each component shows from left to right with level expression frame, which connected with horizontal solid line according to the order of fixed base component, central connecting component, output executive component, this horizontal solid line called component relationship line, relative moving capability relationship ${ }_{i}^{j} M$ written 
below the level component relationship line, $i$ represent the left component on the line, represent the right component on the line. Output executive component and the end component belong to the same one, no expression of relative moving capability between the connected relationship line, description of graph theory of mechanism in Fig. 5 to Fig.7 presented as shown in Fig. 8 to Fig. 10.
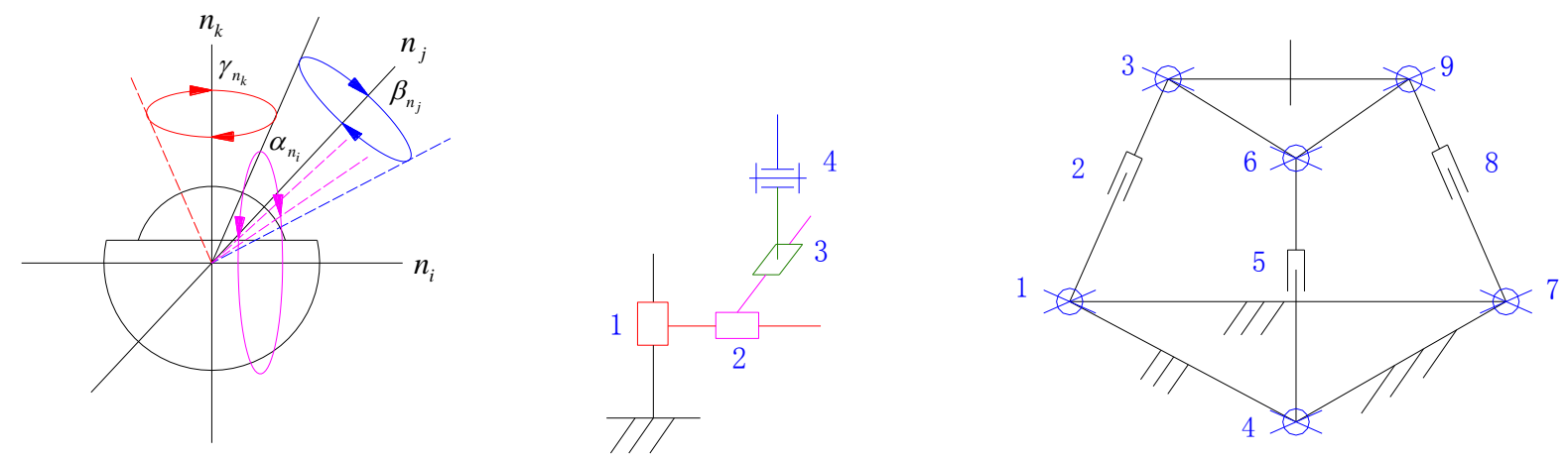

Fig. 4 Spatial moving capability Fig.5 diagram of serial mechanism Fig. 6 diagram of Parallel of sphere joint mechanism

There is only one line of component relationship connected to frame $G$ in the description of serial mechanism (as shown in Figure 8), still only one line of component relationship connected to the left of frame ${ }^{\mathrm{H}}$. Among them, ${ }_{G}^{1} M=\mathrm{P}\left(n_{1}, d_{n_{1}}\right), \quad{ }_{1}^{2} M=\mathrm{P}\left(n_{2}, d_{n_{2}}\right),{ }_{2}^{3} M=\mathrm{P}\left(n_{3}, d_{n_{3}}\right), \quad{ }_{3}^{4} M=\mathrm{P}\left(n_{4}, d_{n_{4}}\right)$.

There are multi lines of component relationship connected to frame $G$ in the description of parallel mechanism (as shown in Figure 9), as well as multi lines of component relationship connected to the left and upper and lower of frame $\mathrm{H}$. Among them, ${ }_{G}^{1} M=\mathrm{T}\left(n_{1}, \alpha_{n_{1}} ; n_{2}, \beta_{n_{2}}\right)$, ${ }_{1}^{2} M=\mathrm{P}\left(n_{3}, d_{n_{3}}\right), \quad{ }_{2}^{3} M=\mathrm{T}\left(n_{4}, \alpha_{n_{4}} ; n_{5}, \beta_{n_{5}}\right), \quad{ }_{\mathrm{G}}^{4} M=\mathrm{T}\left(n_{6}, \alpha_{n_{6}} ; n_{7}, \beta_{n_{7}}\right), \quad{ }_{4}^{5} M=\mathrm{P}\left(n_{8}, d_{n_{8}}\right), \quad{ }_{5}^{6} M=\mathrm{T}\left(n_{9}, \alpha_{n_{9}} ; n_{10}, \beta_{n_{10}}\right)$, ${ }_{\mathrm{G}}^{7} M=\mathrm{T}\left(n_{11}, \alpha_{n_{11}} ; n_{12}, \beta_{n_{12}}\right),{ }_{7}^{8} M=\mathrm{P}\left(n_{13}, d_{n_{13}}\right),{ }_{8}^{9} M=\mathrm{T}\left(n_{14}, \alpha_{n_{14}} ; n_{15}, \beta_{n_{15}}\right)$.

There are multi lines of component relationship connected to frame $\mathrm{G}$ in the description ofhybrid mechanism (as shown in Figure 10), only one solid line of component relationship connected to the left of frame $\mathrm{H}$, which is defined as main component relationship line, still multi dotted lines of component relationship connected to the upper and lower of frame $\bar{B}$, which are defined as constrained component relationship line, frame connected to that also in dotted line, this component is defined as constrained component, constrained component and constrained component relationship line can be any natural number no less than 1 . Among them, ${ }_{\mathrm{G}}^{1} M=\mathrm{P}\left(n_{1}, d_{n_{1}}\right)$, ${ }_{1}^{2} M=\mathrm{P}\left(n_{2}, d_{n_{2}}\right), \quad{ }_{2}^{3} M=\mathrm{P}\left(n_{3}, d_{n_{3}}\right), \quad{ }_{3}^{4} M=\mathrm{T}\left(n_{2}, \alpha_{n_{2}} ; n_{3}, \beta_{n_{3}}\right), \quad{ }_{\mathrm{G}}^{8} M=\mathrm{P}\left(n_{1}, d_{n_{1}}^{\prime}\right), \quad{ }_{8}^{7} M=\mathrm{P}\left(n_{2}, d_{n_{2}}^{\prime}\right), \quad{ }_{7}^{6} M=\mathrm{P}\left(n_{3}, d_{n_{3}}^{\prime}\right)$, ${ }_{6}^{5} M=\mathrm{S}\left(n_{1}, \alpha_{n_{1}}^{\prime} ; n_{2}, \beta_{n_{2}}^{\prime} ; n_{3}, \gamma_{n_{3}}^{\prime}\right)$.

From the above, for any mechanism, it will belong to the category of serial mechanism or parallel mechanism or hybrid mechanism, its description in graph theory can also be expressed as Figure 8 to 10 shown, general expression of arbitrary mechanism can be obtained as figure 11 to 13 shown.
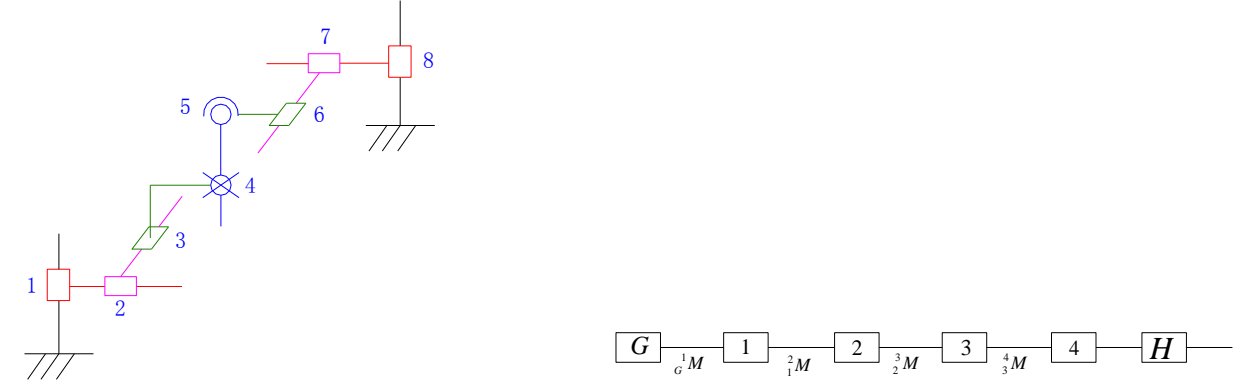

Fig.7 diagram of Hybrid mechanism Fig. 8 description in graph theory of serial mechanism in Fig. 5 


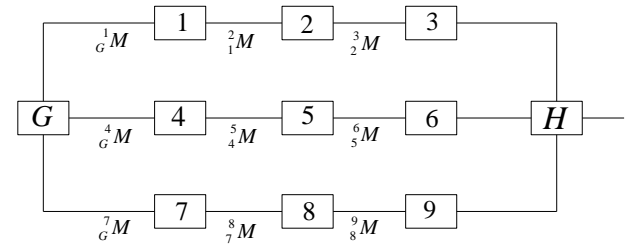

Fig. 9 description in graph theory of parallel mechanism in Fig. 6

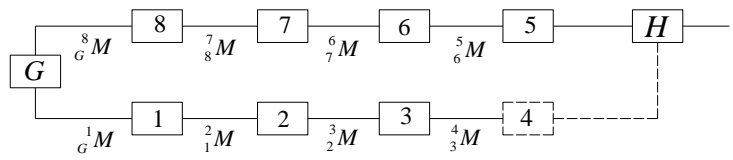

Fig. 10 description in graph theory of hybrid mechanism in Fig. 7

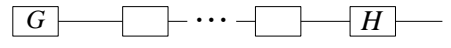

Fig.11 general expression of serial mechanism

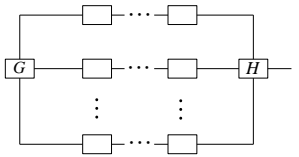

Fig. 12 general expression of parallel mechanism

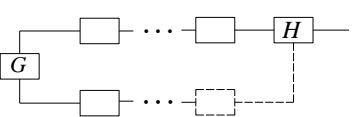

Fig. 13 general expression of hybrid mechanism

\section{Analysis on Working Space and Degree of Freedom of Mechanism}

${ }_{i}^{j} T$ is defined as the conversion matrix of moving coordinate system $T_{j}$ of component ${ }^{j}$ relative to moving coordinate system $T_{j}$ of component $i$. ${ }_{0}^{j} T$ is defined as the conversion matrix of moving coordinate system $T_{j}$ of component $j$ relative to fixed coordinate system $T_{0}$. Spatial relative moving capability relationship ${ }_{i}^{j} M$ of component $j$ relative to component $i$ can be converted to the spatial absolute moving capability ${ }_{0}^{j} M={ }_{0}^{j} T \cdot{ }_{i}^{j} M$ of component ${ }^{j}$ relative to ground bar(fixed coordinate system), relationship ${ }_{0}^{j} T={ }_{0}^{1} T \cdot{ }_{1}^{2} T \cdots{ }_{j-1}^{j} T$ exists between conversion relationship matrix, spatial absolute moving capability formula of component $i$ on arbitrary serial branch relative to fixed coordinate system can be obtained as follow,

$$
{ }_{0}^{i} M={ }_{0}^{1} T \cdot{ }_{1}^{2} T \cdot{ }_{i-1}^{i} T \cdot{ }_{i-1}^{i} M
$$

${ }_{0}^{i} M_{P}^{x},{ }_{0}^{i} M_{P}^{y},{ }_{0}^{i} M_{P}^{z}$ are defined as the components of spatial absolute translation capability ${ }_{0}^{i} M$ along the axis $x, y, z$ in fixed coordinate system, ${ }_{0}^{i} M_{P}$ is the translation component of spatial absolute moving capability ${ }_{0}^{i} M$ of component $i$ relative to fixed coordinate system, following relationship exists between them,

$$
{ }_{0}^{i} M_{P \oplus 0}^{x}{ }_{0}^{i} M_{P}^{y} \oplus{ }_{0}^{i} M_{P=0}^{z}{ }^{i} M_{P},
$$

${ }_{0}^{i} M_{R}^{x},{ }_{0}^{i} M_{R}^{y},{ }_{0}^{i} M_{R}^{z}$ are defined as the units of spatial absolute rotation capability ${ }_{0}^{i} M_{R}$ around the axis $x, y, z$ in fixed coordinate system, ${ }_{0}^{i} M_{R}$ is the rotation unit of spatial absolute moving capability ${ }_{0}^{i} M$ of component $i$ relative to fixed coordinate system, following relationship exists between them,

$$
{ }_{0}^{i} M_{P \oplus 0}^{x}{ }_{0}^{i} M_{P}^{y}{ }_{0}^{i} M_{P=0}^{z}{ }^{i} M_{P},
$$

Therefore, following relationship exists among the translation unit ${ }_{0}^{i} M_{P}$ and the rotation unit ${ }_{0}^{i} M_{R}$ and the spatial absolute moving capability ${ }_{0}^{i} M$ of component $i$ relative to fixed coordinate system, 


$$
{ }_{0}^{i} M_{P \oplus 0_{0}^{i} M_{R=0}{ }^{i} M,},
$$

Suppose there ${ }^{i}$ components exist on the No. $k$ serial branch $S(k)$ of arbitrary mechanism, its spatial absolute moving capability ${ }_{0} S(k)$ relative to fixed coordinate system consists of the spatial absolute moving capability of each component on this serial branch, just as expression 5 to 7 show,

$$
\begin{aligned}
& { }_{0} S(k)_{P}={ }_{0}^{1} M_{P} \oplus_{0}^{2} M_{P} \oplus \cdots \oplus_{0}^{i} M_{P}, \\
& { }_{0} S(k)_{R}={ }_{0}^{1} M_{R} \oplus_{0}^{2} M_{R} \oplus \cdots \oplus_{0}^{i} M_{R}, \\
& { }_{0} S(k)={ }_{0} S(k)_{P} \oplus_{0} S(k)_{R},
\end{aligned}
$$

The units of it meet following relationship,

$$
\begin{aligned}
& { }_{0} S(k)^{x}={ }_{0} S(k)_{P}^{x} \oplus_{0} S(k)_{R}^{x}, \\
& { }_{0} S(k)^{y}={ }_{0} S(k)_{P}^{y} \oplus_{0} S(k)_{R}^{y}, \\
& { }_{0} S(k)^{z}={ }_{0} S(k)_{P}^{z} \oplus_{0} S(k)_{R}^{z}, \\
& { }_{0} S(k)={ }_{0} S(k)^{x} \oplus_{0} S(k)^{y} \oplus_{0} S(k)^{z},
\end{aligned}
$$

As is stated above, the description and operation relations of spatial absolute moving capability of general serial branch or mechanism presented, we can make use of these results to find the description and operation relations of spatial absolute moving capability of general parallel mechanism.

Assume the parallel mechanism consists of two serial branch $k$ and $k+1$, spatial absolute moving capability ${ }_{0} P(k, k+1)$ of output executive component is the intersection of these two branches, thus we have,

$$
{ }_{0} P(k, k+1)={ }_{0} S(k) \cap_{0} S(k+1),
$$

The spatial absolute moving capability of parallel mechanism can also be decomposed into the translation unit and rotation unit, following relationship satisfied then,

$$
\begin{aligned}
& { }_{0} P(k, k+1)={ }_{0} P(k, k+1)_{P} \oplus_{0} P(k, k+1)_{R}, \\
& { }_{0} P(k, k+1)_{P}={ }_{0} S(k)_{P} \cap_{0} S(k+1)_{P}, \\
& { }_{0} P(k, k+1)_{R}={ }_{0} S(k)_{R} \cap_{0} S(k+1)_{R},
\end{aligned}
$$

The spatial absolute moving capability of parallel mechanism and two serial branches can also be decomposed into three translation units along the axis $x, y, z$ and three rotation units around axis $x, y, z$, thus we have,

$$
\begin{aligned}
& { }_{0} P(k, k+1)={ }_{0} P(k, k+1)^{x} \oplus_{0} P(k, k+1)^{y} \oplus_{0} P(k, k+1)^{z}, \\
& { }_{0} P(k, k+1)^{x}=_{0} S(k)^{x} \cap_{0} S(k+1)^{x}, \\
& { }_{0} P(k, k+1)^{y}=_{0} S(k)^{y} \cap_{0} S(k+1)^{y},
\end{aligned}
$$




$$
{ }_{0} P(k, k+1)^{z}={ }_{0} S(k)^{z} \cap_{0} S(k+1)^{z},
$$

Making use of the above method and formula, the general formula and method of spatial absolute moving capability of parallel mechanism with two serial branches obtained, therefore, the general formula of arbitrary parallel mechanism with $k$ serial branches can also be found as following,

$$
\begin{aligned}
& { }_{0} P(1,2, \cdots, k)={ }_{0} S(1) \cap_{0} S(2) \cap \cdots \cap_{0} S(k), \\
& { }_{0} P(1,2, \cdots, k)_{P}={ }_{0} S(1)_{P} \cap_{0} S(2)_{P} \cap \cdots \cap_{0} S(k)_{P}, \\
& { }_{0} P(1,2, \cdots, k)_{R}={ }_{0} S(1)_{R} \cap_{0} S(2)_{R} \cap \cdots \cap_{0} S(k)_{R}, \\
& { }_{0} P(1,2, \cdots k)={ }_{0} P(1,2, \cdots k)^{x} \oplus_{0} P(1,2, \cdots k)^{y} \oplus_{0} P(1,2, \cdots k)^{z},
\end{aligned}
$$

Next, we pay attention to the hybrid mechanism with serial branch and parallel branch, serial branch and parallel branch can be seen as one component unit separately, which connected by a serial component then, its expression and operation relations of spatial absolute moving capability equal to the results of many component connected in series, based on the previous research results, following expressions obtained,

$$
\begin{aligned}
& { }_{0} H={ }_{0} S \oplus_{0} P, \\
& { }_{0} H_{P}={ }_{0} S_{P} \oplus_{0} P_{P}, \\
& { }_{0} H_{R}={ }_{0} S_{R} \oplus_{0} P_{R}, \\
& { }_{0} H={ }_{0} H^{x} \oplus_{0} H^{y} \oplus_{0} H^{z},
\end{aligned}
$$

From the previous research results, spatial absolute moving capability ${ }_{0} W$ of arbitrary spatial mechanism can also be found, which can be written into the summation of translation unit and rotation unit, in addition, which can also be written into the summation of three translation unit along the axis $x, y, z$ and three rotation unit around axis $x, y, z$,

$$
\begin{aligned}
& { }_{0} W={ }_{0} W_{P} \oplus_{0} W_{R}, \\
& { }_{0} W=\left({ }_{0} W_{P}^{x} \oplus_{0} W_{P}^{y} \oplus_{0} W_{P}^{z}\right) \oplus\left({ }_{0} W_{R}^{x} \oplus_{0} W_{R}^{y} \oplus_{0} W_{R}^{z}\right),
\end{aligned}
$$

From expression 29, we have intuitive results of working space of mechanism, degree of freedom of mechanism can also be judged conveniently from the number of its component unit.

\section{Inverse Design of Mechanism}

When given the target task, spatial moving capability range(large, middle, small) and requirement of accuracy(high, middle, low) and loading capability(large, middle, small) of output executive end have been known to us, according to the task requirements to design the most simple and effective mechanism is the process of inverse design, make use of the above results, take the inverse design of mechanism with single moving capability for example, as shown in Table 1, select serial or parallel mechanism according to the level of working space and precision and loading capability, $\mathrm{S}\left(^{*}\right)$ means serial mechanism meet the requirements, $\mathrm{P}(*)$ means parallel mechanism meet the requirements. The recommendation criterion includes select serial mechanism when the number of $\mathrm{S}\left({ }^{*}\right)$ equal to 3 , select hybrid mechanism when the number of $\mathrm{S}\left(^{*}\right)$ equal to 2 , select parallel or hybrid mechanism when the number of $S\left(^{*}\right)$ equal to 1 , select parallel mechanism when the number 
of $\mathrm{S}(*)$ equal to 0 . The judging condition expressed with $\mathrm{A}$ and $\mathrm{B}$ and $\mathrm{C}$ in behalf of large or high and middle and small or low, S, P, H represents the serial, parallel, hybrid, the recommendation mechanism type can also be determined by the number of $\mathrm{P}(*)$.

Tab. 1 Inverse design of mechanism with single moving capability

\begin{tabular}{|c|c|c|c|c|c|c|}
\hline $\begin{array}{l}\text { Moving } \\
\text { capability } \\
\text { type }\end{array}$ & $\begin{array}{l}\text { Pair } \\
\text { type }\end{array}$ & $\begin{array}{l}\text { Working space } \\
\text { level }\end{array}$ & $\begin{array}{l}\text { Accuracy } \\
\text { level }\end{array}$ & $\begin{array}{l}\text { loading } \\
\text { capability } \\
\text { level }\end{array}$ & Judging process & $\begin{array}{l}\text { Recommended } \\
\text { mechanism type }\end{array}$ \\
\hline \multirow{27}{*}{ translation } & \multirow{27}{*}{$1 \mathrm{P}$} & \multirow{9}{*}{ A } & \multirow{3}{*}{ A } & $\bar{A}$ & $\mathrm{~S}(\mathrm{~A}) \cup \mathrm{P}(\mathrm{A}) \cup \mathrm{P}(\mathrm{A})$ & $\mathrm{P} / \mathrm{H}$ \\
\hline & & & & B & $S(A) \cup P(A) \cup S(B)$ & $\mathrm{H}$ \\
\hline & & & & $\mathrm{C}$ & $\mathrm{S}(\mathrm{A}) \cup \mathrm{P}(\mathrm{A}) \cup \mathrm{S}(\mathrm{C})$ & $\mathrm{H}$ \\
\hline & & & \multirow{3}{*}{ B } & A & $\mathrm{S}(\mathrm{A}) \cup \mathrm{S}(\mathrm{B}) \cup \mathrm{P}(\mathrm{A})$ & $\mathrm{H}$ \\
\hline & & & & B & $\mathrm{S}(\mathrm{A}) \cup \mathrm{S}(\mathrm{B}) \cup \mathrm{S}(\mathrm{B})$ & $\mathrm{S}$ \\
\hline & & & & $\mathrm{C}$ & $S(A) \cup S(B) \cup S(C)$ & $\mathrm{S}$ \\
\hline & & & \multirow{3}{*}{$\mathrm{C}$} & $\mathrm{A}$ & $\mathrm{S}(\mathrm{A}) \cup \mathrm{S}(\mathrm{C}) \cup \mathrm{P}(\mathrm{A})$ & $\mathrm{H}$ \\
\hline & & & & $\mathrm{B}$ & $S(A) \cup S(C) \cup S(B)$ & $\mathrm{S}$ \\
\hline & & & & $\mathrm{C}$ & $S(A) \cup S(C) \cup S(C)$ & $\mathrm{S}$ \\
\hline & & \multirow{9}{*}{ B } & \multirow{3}{*}{ A } & A & $S(B) \cup P(A) \cup P(A)$ & $\mathrm{P} / \mathrm{H}$ \\
\hline & & & & B & $S(B) \cup P(A) \cup S(B)$ & $\mathrm{H}$ \\
\hline & & & & $\mathrm{C}$ & $S(B) \cup P(A) \cup S(C)$ & $\mathrm{H}$ \\
\hline & & & \multirow{3}{*}{ B } & A & $S(B) \cup S(B) \cup P(A)$ & $\mathrm{H}$ \\
\hline & & & & $\mathrm{B}$ & $S(B) \cup S(B) \cup S(B)$ & $\mathrm{S}$ \\
\hline & & & & $\mathrm{C}$ & $S(B) \cup S(B) \cup S(C)$ & $\mathrm{S}$ \\
\hline & & & \multirow{3}{*}{$\mathrm{C}$} & A & $S(B) \cup S(C) \cup P(A)$ & $\mathrm{H}$ \\
\hline & & & & B & $S(B) \cup S(C) \cup S(B)$ & $\mathrm{S}$ \\
\hline & & & & $\mathrm{C}$ & $S(B) \cup S(C) \cup S(C)$ & $\mathrm{S}$ \\
\hline & & \multirow{9}{*}{$\mathrm{C}$} & \multirow{3}{*}{ A } & A & $S(C) \cup P(A) \cup P(A)$ & $\mathrm{P} / \mathrm{H}$ \\
\hline & & & & B & $\mathrm{S}(\mathrm{C}) \cup \mathrm{P}(\mathrm{A}) \cup \mathrm{S}(\mathrm{B})$ & $\mathrm{H}$ \\
\hline & & & & $\mathrm{C}$ & $S(C) \cup P(A) \cup S(C)$ & $\mathrm{H}$ \\
\hline & & & \multirow{3}{*}{ B } & A & $S(C) \cup S(B) \cup P(A)$ & $\mathrm{H}$ \\
\hline & & & & B & $S(C) \cup S(B) \cup S(B)$ & $\mathrm{S}$ \\
\hline & & & & $\mathrm{C}$ & $S(C) \cup S(B) \cup S(C)$ & $\mathrm{S}$ \\
\hline & & & \multirow{3}{*}{$\mathrm{C}$} & A & $S(C) \cup S(C) \cup P(A)$ & $\mathrm{H}$ \\
\hline & & & & B & $S(C) \cup S(C) \cup S(B)$ & $\mathrm{S}$ \\
\hline & & & & $\mathrm{C}$ & $S(C) \cup S(C) \cup S(C)$ & $\mathrm{S}$ \\
\hline
\end{tabular}

From the above, there are types of 2P, 3P, 1P1R, 1P2R, 1P3R, 2P1R, 2P2R, 2P3R, 3P1R, 3P2R, 3P3R except $1 \mathrm{P}$ target moving capability in mechanism design, there are three hundreds and twenty four types of mechanisms in total, method is the same as shown in Table 1, that will not described here.

\section{Conclusion}

New analysis and design method obtained based on the previous research on graph theory of mechanism, following conclusions found then.

(1) New description method in graph theory based on components and relative spatial moving capability between components presented, it can meet the effective description for serial, parallel and hybrid mechanism.

(2) Dimensionality of spatial moving capability of branch and mechanism defined based on the graph theory and motion base, analysis method and intuitive description of serial and parallel and hybrid mechanism obtained then.

(3) Make use of twelve types of classification of target spatial moving capability of output end, the level of working space and precision and loading capability selected as the design conditions, still judging methods and criteria listed here, there will three hundreds and twenty four types of mechanism recommended in all. 


\section{References}

[1] F. Freudenstein, L. Dobrjanskyj. On a theory of type synthesis of mechanisms: submitted to Journal of Applied Mechanic (1964).

[2] L. K. Deng. Graph theory and its application]. Tsinghua University Publishing House, Beijing, 1984.

[3] D. H. Feng, H. Zhen. Automatic creation of topological graphs and the characteristic representations of kinematic chains based on the loop characteristics: submitted to Journal of Mechanical Engineering(2007).

[4] M. Grübler. Allgemeine Eigenschaften der zwangläufigen ebenen kinematische kette: I, Civilingenieur, 1883.

[5] M. Grübler Allgemeine Eigenschaften der zwangläufigen ebenen kinematische kette: II, Verein zur Beforderung des Gewerbefleisses. Verhandlungen, 1885.

[6] K. Kutzbach, Einzelfragen aus dem gebiet der Maschinenteile, Zeitschrift der Verein Destscher Ingenieur( 1933).

[7] C. H. Suh, C. W. Radcliffe. Kinematics and Mechanisms Design. John Wiley\&Sons, Inc, New York, 1978.

[8] G. N. Sandor, A. G. Erdman. Advance Mechanism: Analysis and Synthesis. Englewood Cliffs, Prentice Hall, 1984.

[9] H. Zhen, Z. Y. Sheng, Z. T. Shi. High level spacial mechanics. High Education Publishing House, Beijinig, 2006.

[10]Y. T. Li. Basic theory on configuration and kinematics and kinetics of machinery system. Machinery Industry Publishing House, Beijing, 1995. 